http://dx.doi.org/10.11646/zoosymposia.14.1.18

http://zoobank.org/urn:Isid:zoobank.org:pub:2B1F3DDD-1C64-4EF3-9884-6354CBFAF1ED

\title{
Caddisflies collected using a Malaise trap at a spring-fed brook of Shimauchi-yusui in the Matsumoto Basin, central Japan: fauna and phenology
}

\author{
TAKAO NOZAKI ${ }^{1, *}$, TOMIKO ITO ${ }^{2} \&$ KOJI TOJO ${ }^{3,4}$ \\ 13-16-15, Midorigaoka, Ninomiya-machi, Naka-gun, Kanagawa 259-0132, Japan.E-mail: takao.nozaki@nifty.com \\ ${ }^{2}$ Hokkaido Aquatic Biology, Hakuyo-cho, 3-3-5, Eniwa, Hokkaido 061-1434, Japan \\ ${ }^{3}$ Department of Biology, Faculty of Science, Shinshu University, Asahi 3-1-1, Matsumoto, Nagano 390-8621, Japan \\ ${ }^{4}$ Institute of Mountain Science, Shinshu University, Asahi 3-1-1, Matsumoto, Nagano 390-8621, Japan \\ *corresponding author
}

\begin{abstract}
Adult caddisflies were collected weekly from a spring-fed brook of Shimauchi-yusui from 11 April 2013 to 5 June 2014. A total of 11867 specimens belonging to 39 species, 18 genera and 14 families were identified. The most abundant species collected in 1 year (the first 52 weeks) were Agapetus sibiricus Martynov 1918 (60\%), Apatania aberrans (Martynov 1933) (12\%) and Micrasema spinosum Nozaki and Tanida 2007 (5.4\%). The Trichoptera fauna mainly reflected the major larval habitats of this brook, but other factors such as water temperature also probably affect species composition. The most common species (wherein more than 50 individuals were collected) had discrete seasonal flight periods.
\end{abstract}

Key words: spring-fed stream, adult Trichoptera, species composition, flight period, seasonality

\section{Introduction}

Springs and spring-fed brooks are unique freshwater habitats, and their ecology and faunal assemblages, including the Trichoptera, have been studied by many researchers (e.g., Botosaneanu 1998). In Japan, however, investigations on caddisflies in such environments are scarce, although there are plenty of springs. In Hokkaido, northern Japan, the Trichopteran fauna were studied using Malaise traps from three cold springfed streams, and flight periods of several species were reported (Ito et al. 1998, Ohkawa 1999, Kuhara 2011). Nozaki and Tanida (2007) and Nozaki et al. (2016) also reported on the Trichopteran fauna of two spring-fed streams in central Honshu based on Malaise trap collection.

The Matsumoto Basin is situated in central Honshu and is flanked by 2000-3000 m mountain ranges that supply underground water to many springs in the lower fan zone (Fig. 1). Most springs and spring-fed streams are used for domestic water, and some are also used for the cultivation of plants (mainly wasabi) and fish, such as trout. However, knowledge about the fauna, especially the invertebrates, of these spring-fed streams is very poor. The Shimauchi-yusui is a spring-fed stream in this area. It is supplemented with water from two spring-fed brooks that emerge from the left river terrace of the Narai River. It flows for approximately $1 \mathrm{~km}$ to its confluence with the Narai River. These brooks are maintained by local residents who keep the water clean. We studied adult caddisflies using a Malaise trap set at one of the brooks for collection. Here we report on the caddisfly fauna of this spring-fed brook and the phenology of their flight period. 


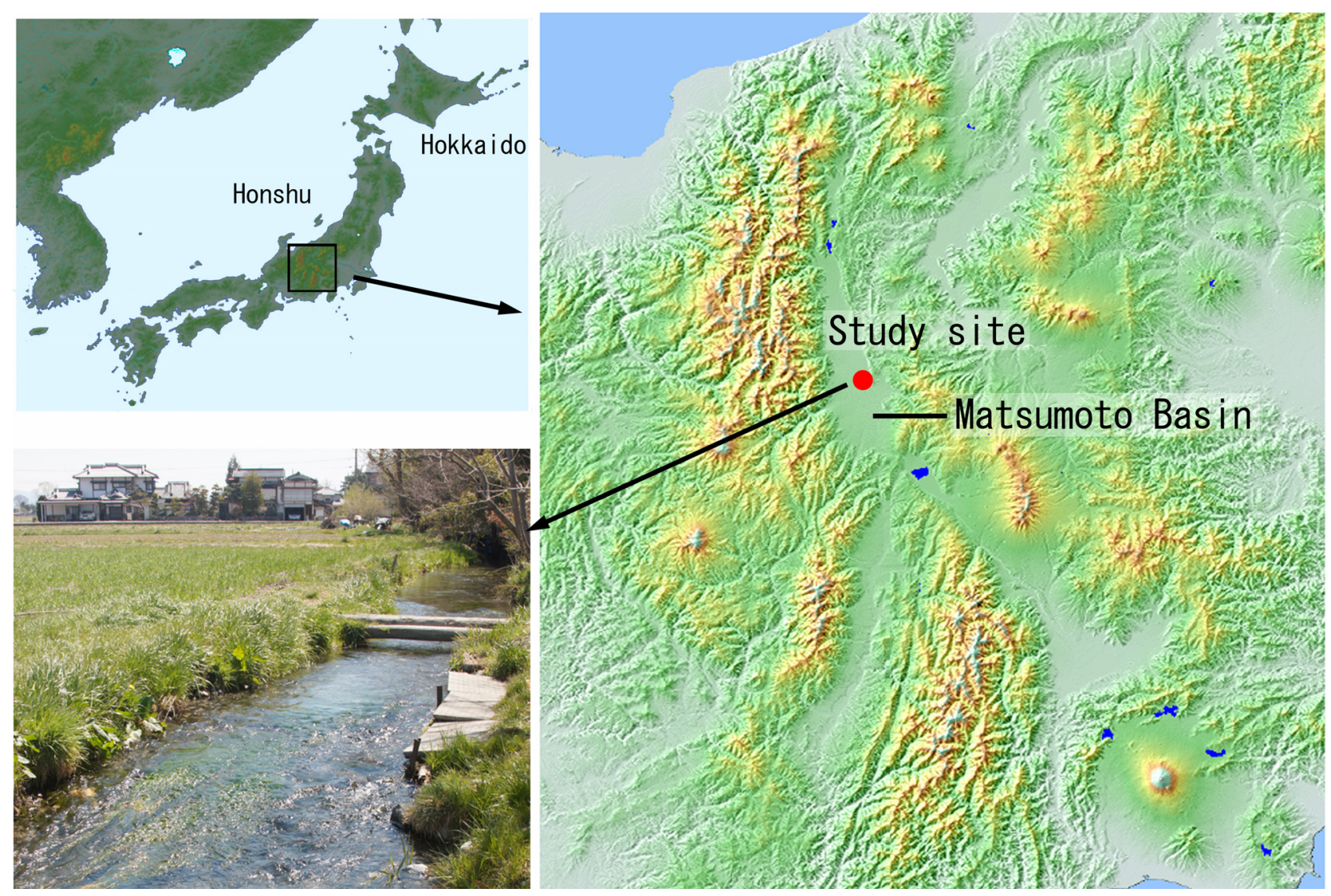

FIGURE 1. Location and photograph of the study site.

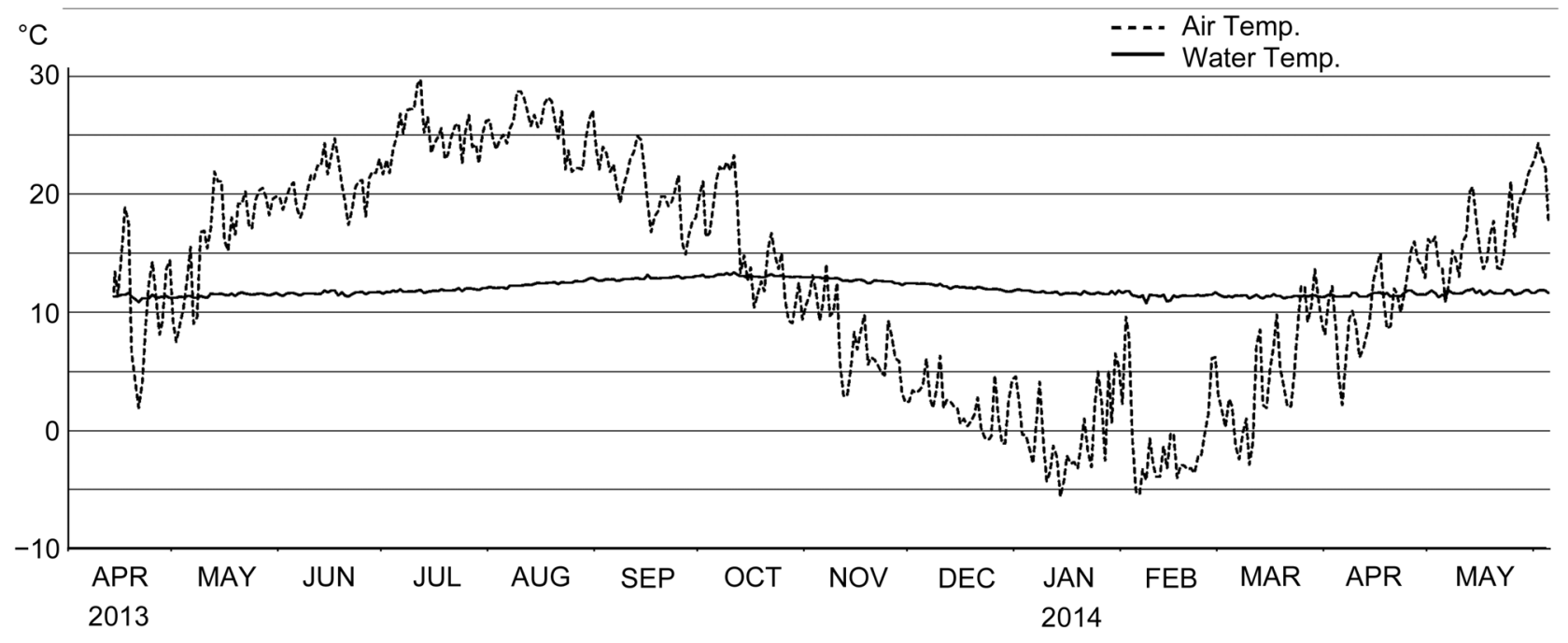

FIGURE 2. Changes in average daily water and air temperatures. Air temperature data were obtained from the Matsumoto meteorological station.

\section{Materials and methods}

The study site $\left(36^{\circ} 15^{\prime} 24.6^{\prime \prime} \mathrm{N}, 137^{\circ} 56^{\prime} 42.3^{\prime \prime}, 570 \mathrm{~m}\right.$ a.s.l.) is approximately $300 \mathrm{~m}$ from the spring source, and the brook flows between a paddy field and a forested slope (Fig. 1). The width of the channel was 
approximately $200 \mathrm{~cm}$, the water depth approximately $50 \mathrm{~cm}$, and the current speed approximately $40 \mathrm{~cm} / \mathrm{s}$ on average. The stream bed was dominated by cobbles and gravels and had a rich assemblage of aquatic plants (Veronica anagallis-aquatica, Ranunculus nipponicus, and Potamogeton crispus) and bryophytes (Chiloscyphus polyanthos and Rhynchostegium riparioides). The channel side walls were constructed of stone masonry. The annual average water temperature based on daily averages measured using a temperature logger (Hobo TidbiT ${ }^{\circledR} \mathrm{v} 2$, Onset Computer cooperation) from 12 April 2013 to 11 April 2014 was $12.0^{\circ} \mathrm{C}$ (range, $10.8-13.4^{\circ} \mathrm{C}$ ) (Fig. 2). The air temperature recorded at the Matsumoto meteorological station (approximately $2.5 \mathrm{~km}$ to the southeast of the study site) during the same period was $12.3^{\circ} \mathrm{C}$ (range, $-5.6-29.7^{\circ} \mathrm{C}$ ) (Japan Meteorological Agency 2014) (Fig. 2).

Adult caddisflies were collected weekly using a Townes-style Malaise trap (Townes 1972) from 11 April 2013 to 5 June 2014. All specimens were preserved in 80\% ethyl alcohol and deposited in the personal collections of T. Ito (Hydroptilidae and Lepidostomatidae) and T. Nozaki (others).

\section{List of caddisflies}

Rhyacophilidae

Rhyacophila brevicephala Iwata 1927

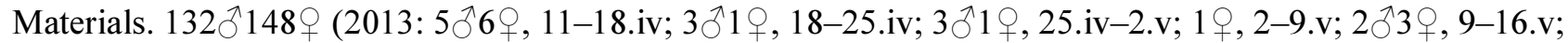

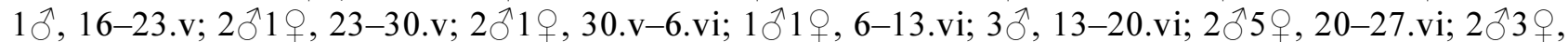

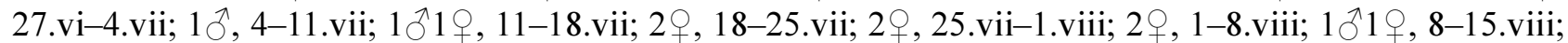

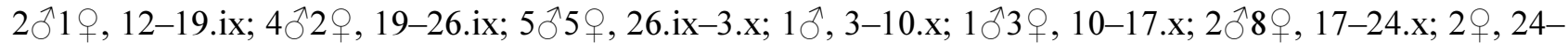

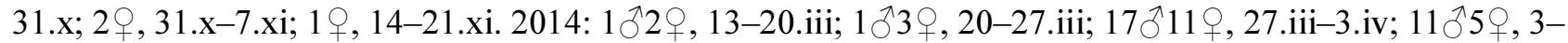

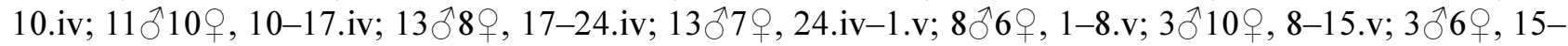

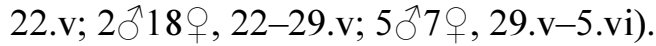

Rhyacophila kuwayamai Schmid 1970

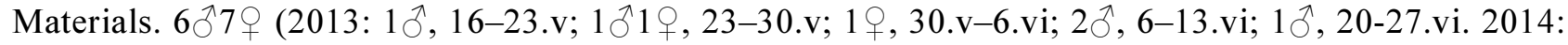
$1{ }^{\jmath} 2$ \% , 22-29.v; $3+$, 29.v-5.vi).

Rhyacophila nigrocephala Iwata 1927

Material. $1 \widehat{\jmath}(2013: 1 \jmath$, 27.vi-4.vii).

Rhyacophila nipponica Navás 1933

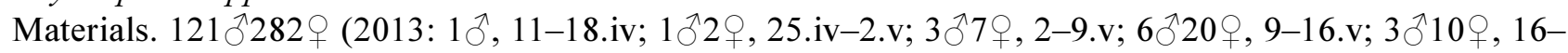

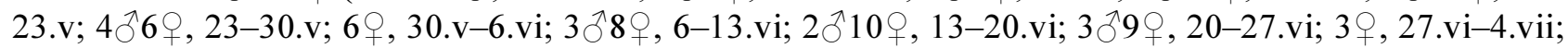

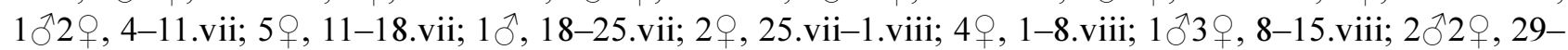

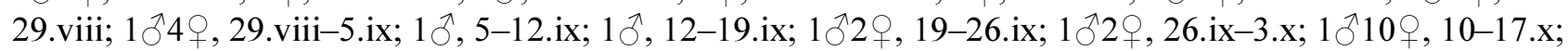

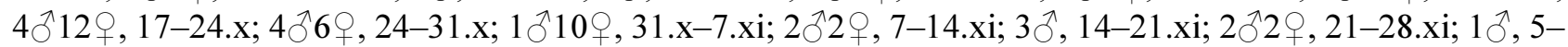

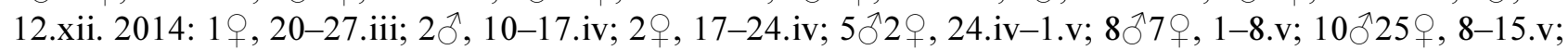

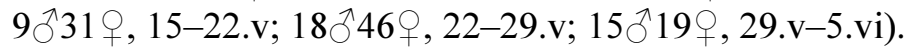

Rhyacophila yamanakensis Iwata 1927

Materials. $2 \overbrace{}^{\lambda} 1 q$ (2013: 1q, 6-13.vi. 2014: 2ð, 24.iv-1.v).

Hydroptilidae

Hydroptila botosaneanui Kumanski 1990

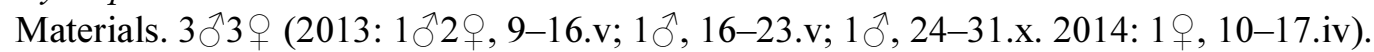


Hydroptila phenianica Botosaneanu 1970

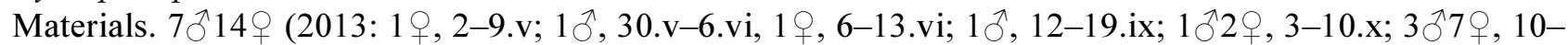
17.x; 1, 17-24.x. 2014: 1ㅇ, 27.iii-3.iv; 1ठ, 3-10.iv; 1ㅇ, 24.iv-1.v).

Oxyethira angustella Martynov, 1933

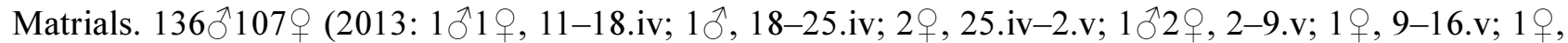

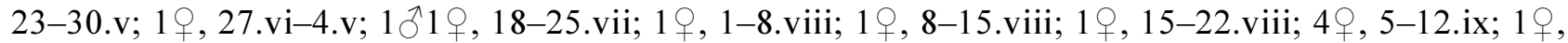

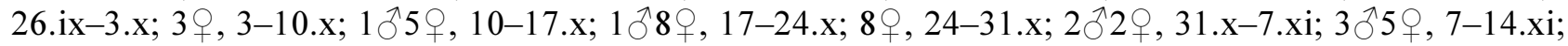

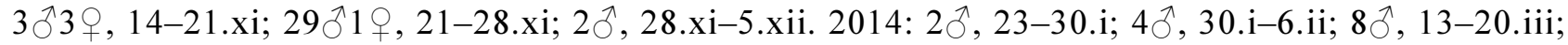

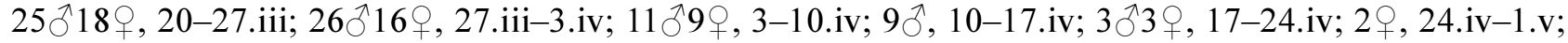
$3{ }^{\lambda} 6$ ㅇ, 8-15.v; 1 우 $\left.15-22 . v\right)$.

Glossosomatidae

Agapetus sibiricus Martynov 1918

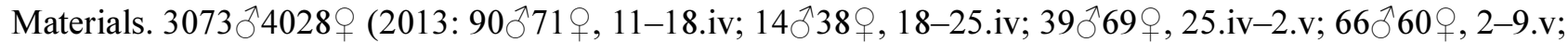

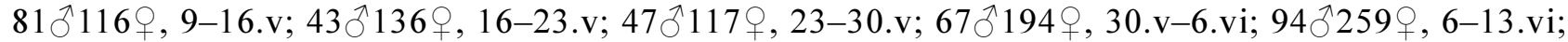

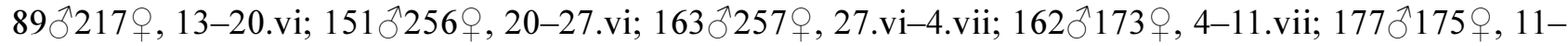

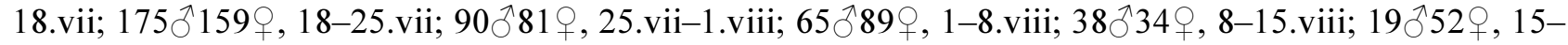

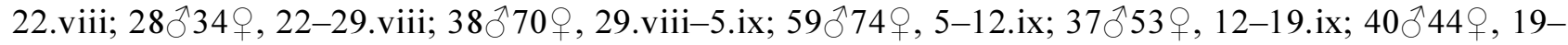

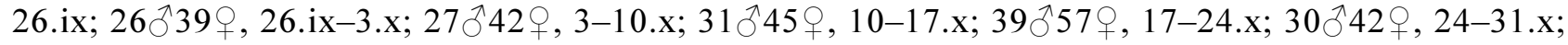

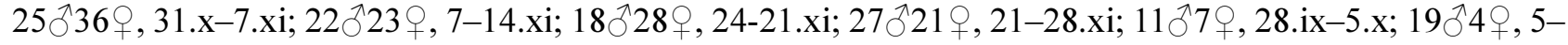

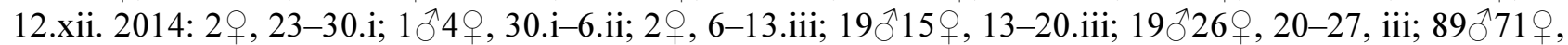

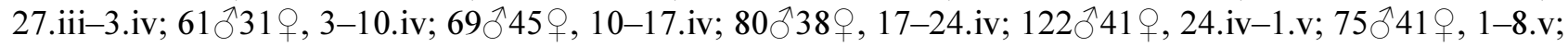

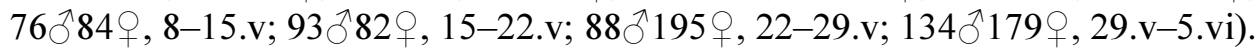

Glossosoma altaicum (Martynov 1914)

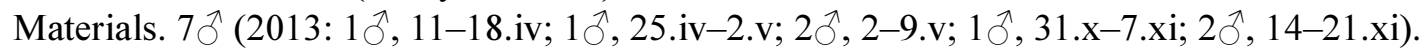

Glossosoma ussuricum (Martynov 1934)

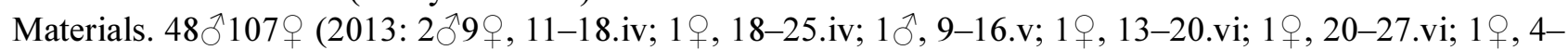

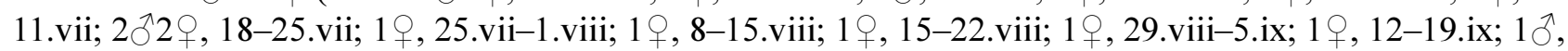

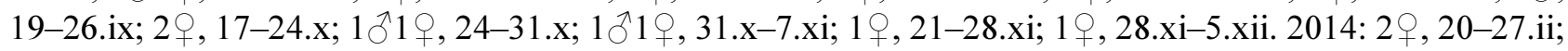

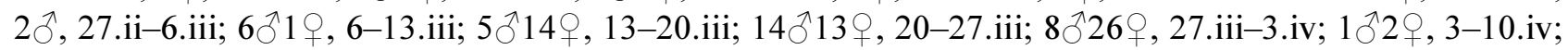
$3 \widehat{\jmath} 15$ ㅇ, 10-17.iv; 4ㅇ, 17-24.iv; 1 ภ우, 24.iv-1.v).

\section{Stenopsychidae}

Stenopsyche marmorata Navás 1920

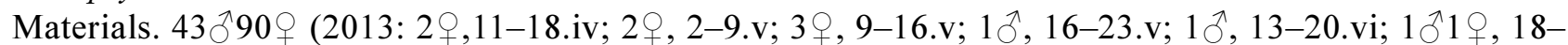

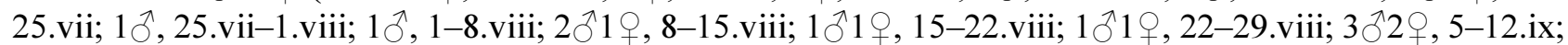

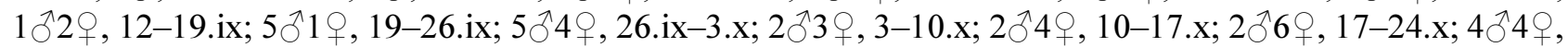

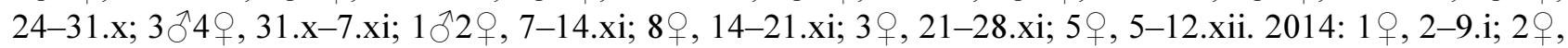

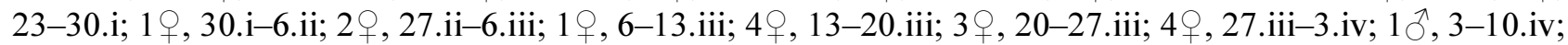

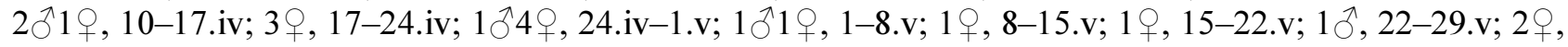
29.v-5.vi).

\section{Psychomyiidae}

Psychomyia acutipennis (Ulmer 1908)

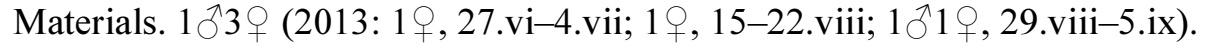


Psychomyia armata Schmid 1964

Material. $1 \hat{\jmath}(2013: 1 \hat{\jmath}, 29 . v i i i-5 . i x)$.

Psychomyia morisitai Tsuda 1942

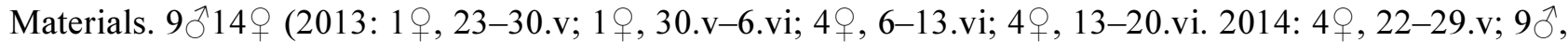
29.v-5.vi).

Tinodes higashiyamanus Tsuda 1942

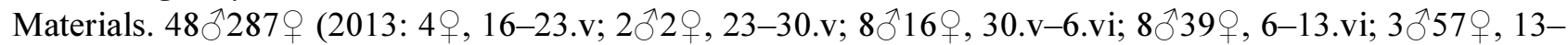

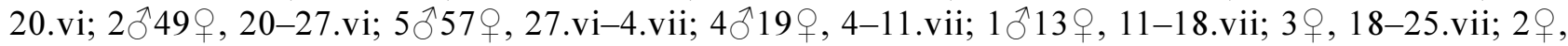
25.vii-1.viii; 1 ㅇ, 1-8.viii; 1 ㅇ, 8-15.viii; 3 ㅇ, 22-29.viii; 3ㅇ, 29.viii-5.ix; 1 ㅇ, 5-12.ix. 2014: 1, 22-29.v; $8 \curvearrowright 18$ ค , 29.v-5.vi).

Tinodes sp. (aff. miyakonis Tsuda 1942)

Material. 1ठ(2013: 1へ, 6-13.vi).

Hydropsychidae

Cheumatopsyche brevilineata (Iwata 1927)

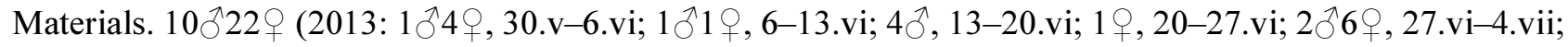

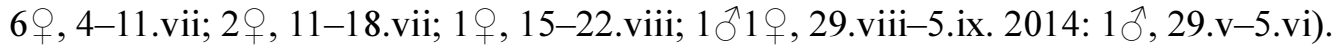

Cheumatopsyche infascia Martynov 1934

Materials. 4 ㅇ (2013: 1, 20-27.vi; 2ㅇ, 27.vi-4.vii; 1, 4-11.vii).

Hydropsyche orientalis Martynov 1934

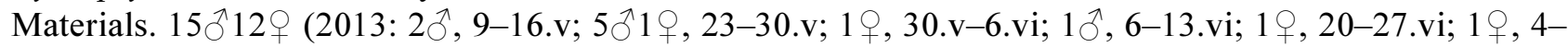

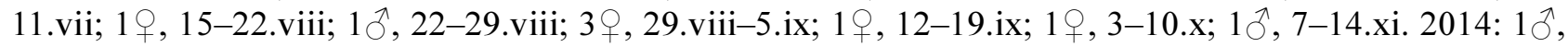

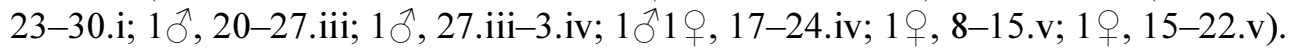

Hydropsyche setensis Iwata 1927

Material. 1 ㅇ $(2013: 1$,, $23-30 . v)$.

Phryganopsychidae

Phryganopsyche latipennis (Banks 1906)

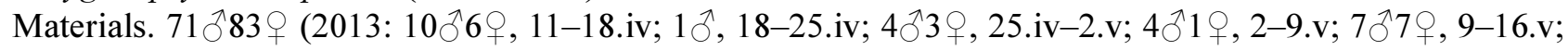

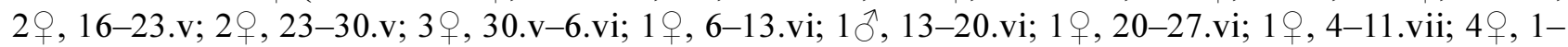

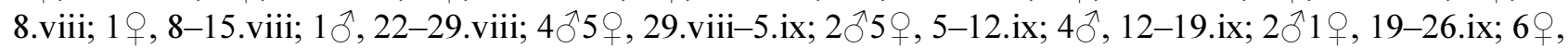
26.ix-3.x; 3ㅇ, 3-10.x; 1ㅇ, 10-17.x; $2^{\Uparrow} 5$ ㅇ, 17-24.x; 5ภ, 24-31.x; $1 \jmath^{\lambda}, 31 . x-7 . x i$; $1{ }^{\lambda} 2$ ㅇ, 14-21.xi; $1 \jmath^{\lambda}, 5-$

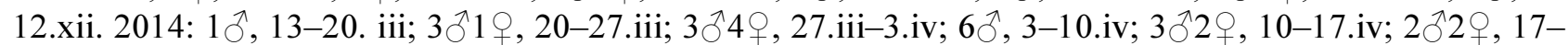

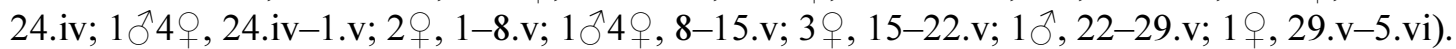

Brachycentridae

Micrasema akagiae Nozaki and Tanida 2007

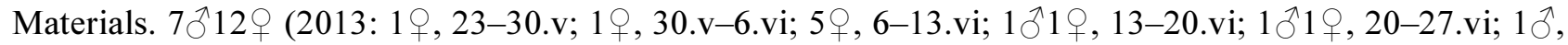

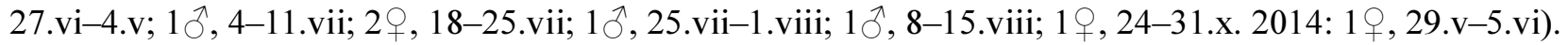

Micrasema hanasense Tsuda 1942

Material. 1 ㅇ (2013: 1우, 20-27.vi). 
Micrasema spinosum Nozaki and Tanida 2007

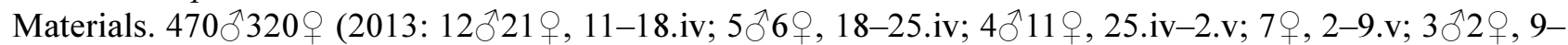

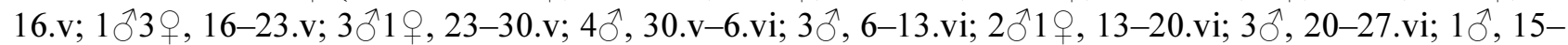

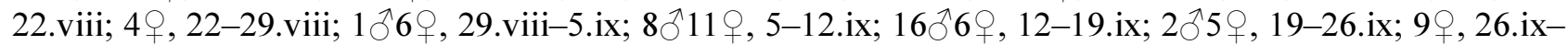

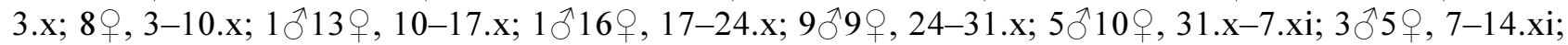

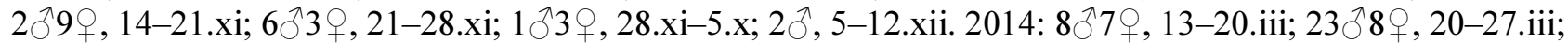

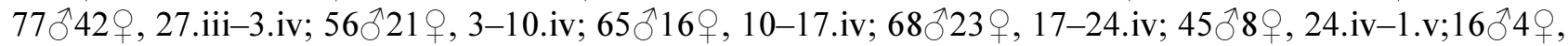

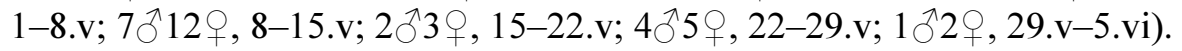

\section{Lepidostomatidae}

Lepidostoma bipertitum (Kobayashi 1955)

Material. 1 ㅇ (2013: 1ㅇ, 2-9.v).

Lepidostoma japonicum (Tsuda 1936)

Material. 1 ㅇ (2013: 1, 24-31.x).

Lepidostoma kojimai (Tani 1971)

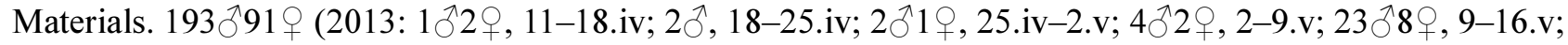

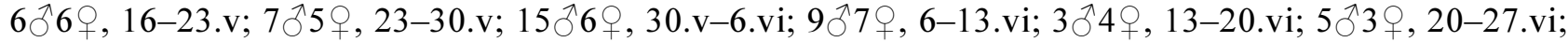

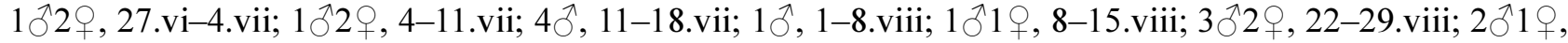

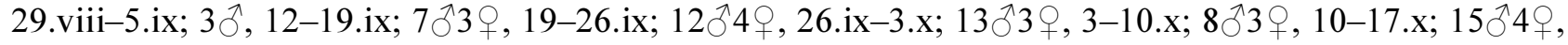

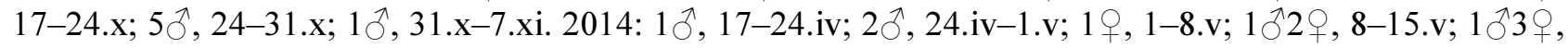

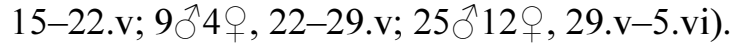

Lepidostoma orientale (Tsuda 1942)

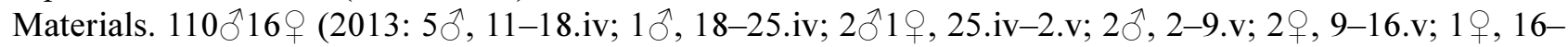
23.v; 1, 6-13.vi; 1, 12-19.ix; 1ð2

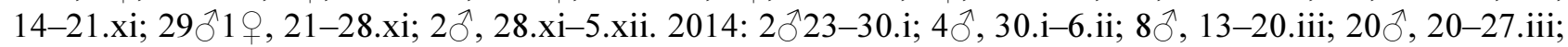

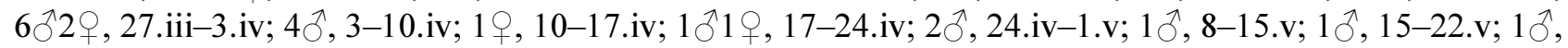
22-29.v).

Lepidostoma satoi (Kobayashi 1968)

Material. $1 \widehat{\jmath}(2014: 1 \hat{\jmath}, 8-15 . v)$.

\section{Limnephilidae}

Nothopsyche pallipes Banks 1906

Material. 1 + $(2013: 1+$, 17-24.x).

Nothopsyche ruficollis (Ulmer 1905)

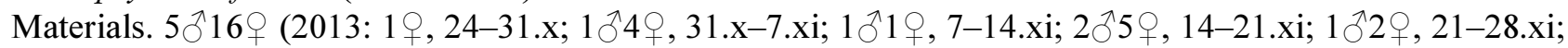
2 , 28.xi-5.xii; $19,5-12 . x i i)$.

Nothopsyche ulmeri Schmid 1952

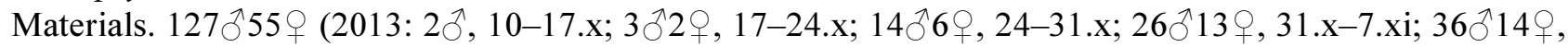

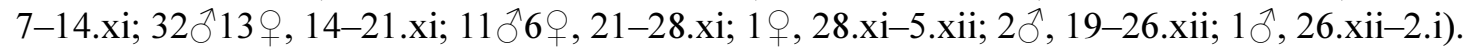


Apataniidae

Apatania aberrans (Martynov 1933)

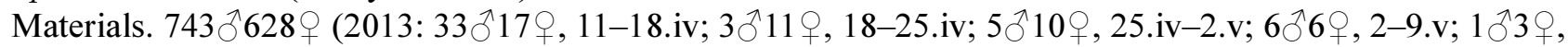

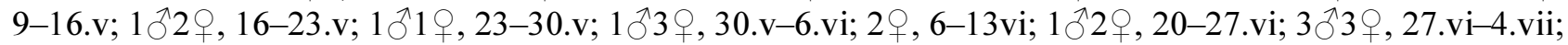

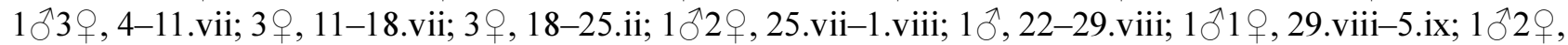

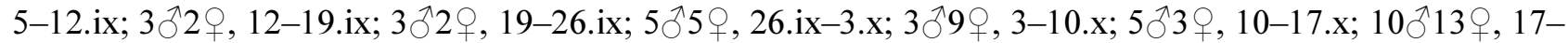

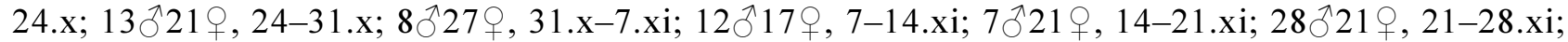

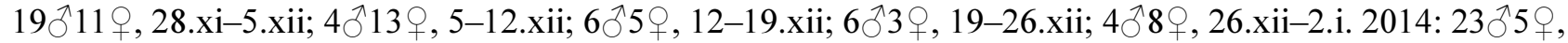

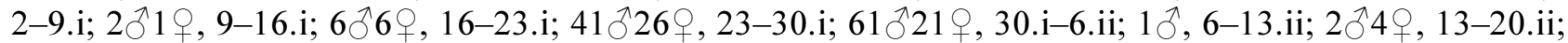

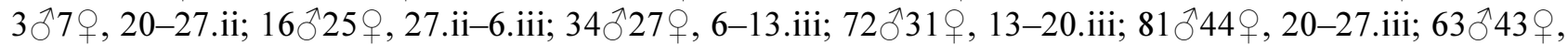

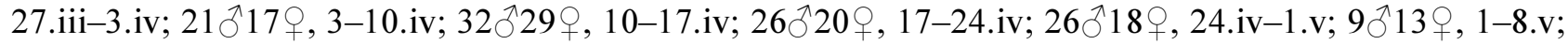

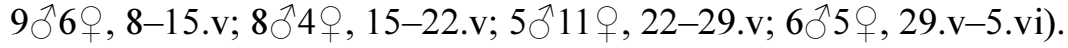

Apatania kyotoensis Tsuda 1939

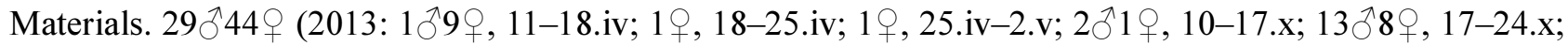

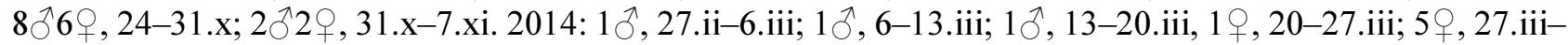
3.iv; 4ㅇ, 3-10.iv; 5ㅇ, 10-17.iv; 1ㅇ, 17-24.iv).

Apatania nikkoensis Tsuda 1939

Material. 1 ㅇ (2013: 1, 21-28.xi).

Goeridae

Goera japonica Banks 1906

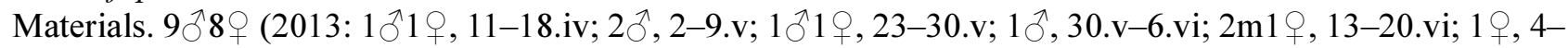

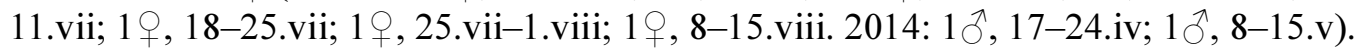

\section{Leptoceridae}

\section{Oecetis nigropunctata Ulmer 1908}

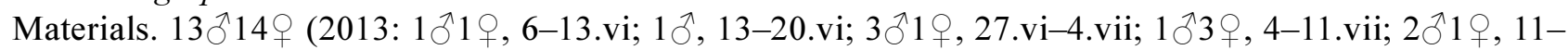

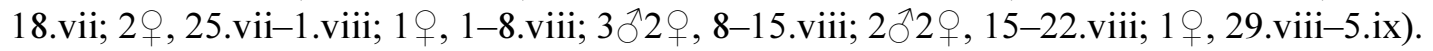

Sericostomatidae

Gumaga orientalis (Martynov 1935)

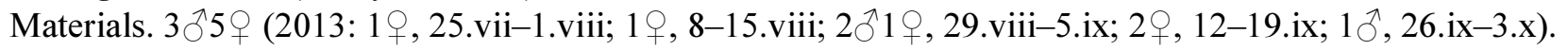

\section{Trichoptera fauna of Shimauchi-yusui}

A total of 11867 specimens belonging to 39 species, 18 genera and 14 families were recorded from this spring-fed brook. The most abundant species collected in 1 year (the first 52 weeks) were Agapetus sibiricus (60\%), Apatania aberrans (12\%), and Micrasema spinosum (5.4\%) (Table 1). A. sibiricus and A. aberrans larvae were abundant on stream bed cobbles and stone retaining walls of the channel, whereas $M$. spinosum was more abundant on bryophytes. These abundant species apparently reflect the preferential larval habitats in this stream. 
TABLE 1. Comparison between the average water temperatures, major larval habitats, and three abundant species from three spring-fed streams in central Honshu, Japan. The number in parenthesis shows the proportion (\%) of each species relative to the total number in 1 year. Data from the Kakida River and Jimotoyusui were obtained from Nozaki \& Tanida (2007) and Nozaki et al. (2016), respectively.

\begin{tabular}{llll}
\hline & Shimauchi-yusui & Kakida River & Jimoto-yusui \\
\hline Average water temperature & $12^{\circ} \mathrm{C}$ & $15^{\circ} \mathrm{C}$ & $11.4^{\circ} \mathrm{C}$ \\
Major larval habitats & Cobbles \& gravels, aquatic & Sand bed, aquatic plants, & Plant debris, roots of riparian \\
& plants, bryophytes & mosses & vegetation \\
Abundant species & Agapetus sibiricus (60) & Micrasema akagiae (45) & Lepidostoma kanbaranum (48) \\
& Apatania aberrans (12) & Leptocerus fluminalis* (24) & Oecetis nigropunctata (16) \\
& Micrasema spinosum (5.4) & Gumaga orientalis (8) & Philocentropus shigae (13) \\
\hline
\end{tabular}

*: recorded as Leptocerus sp. in the original paper.

In central Honshu, the Trichopteran fauna of the Kakida River and Jimoto-yusui using the same method as that in this study has been reported (Nozaki and Tanida 2007 and Nozaki et al. 2016). The Trichoptera species composition in the present study differs from these other two spring-fed streams (Tables 1, 2). Considering that the abundant species of the Kakida River and Jimoto-yusui also reflected their larval habitats, these habitats must be an important factor that characterizes the caddisfly fauna of each spring-fed stream. However, although the Shimauchi-yusui is rich in aquatic plants, species associated with this habitat were not abundant. Leptocerus fluminalis Ito and Kuhara 2009 inhabit aquatic plant assemblages in the larval stage and was the second most abundant species in the Kakida River (Table 1), but this species was not collected from our study site. Furthermore, although three Micrasema species-M. akagiae, M. hanasense, and $M$. spinosum - were found in both the Shimauchi-yusui and Kakida River, their dominance varied among streams (Table 1). In the Shimauchi-yusui, M. spinosum was the most abundant of the three species (96.4\%) followed by $M$. akagiae (3.4\%), but in the Kakida River, M. akagiae was the most abundant species $(95.5 \%)$ followed by $M$. hanasense (4.2\%). These results suggest that other factors, such as the lower water temperature of the Shimauchi-yusui compared with that of the Kakida River, also affect species composition.

TABLE 2. Similarity matrix of Trichoptera communities from three spring-fed streams in central Honshu, Japan [Morishita's Similarity Index C $\lambda$ (Morishita 1959)]. Data from the Kakida River and Jimoto-yusui were obtained from Nozaki \& Tanida (2007) and Nozaki et al. (2016), respectively.

\begin{tabular}{lcc}
\hline & Kakida River & Jimoto-yusui \\
\hline Shimauchi-yusui & 0.011 & 0.011 \\
Kakida River & - & 0.000 \\
\hline
\end{tabular}

\section{Flight period of Trichoptera adults}

Figures 3 and 4 show the seasonal occurrence of 14 species, of which more than 50 individuals of each species were collected. Most species had a discrete seasonal flight period, and these data provide information about their emergence season and voltinism, although precise life cycle studies are needed for confirmation. 

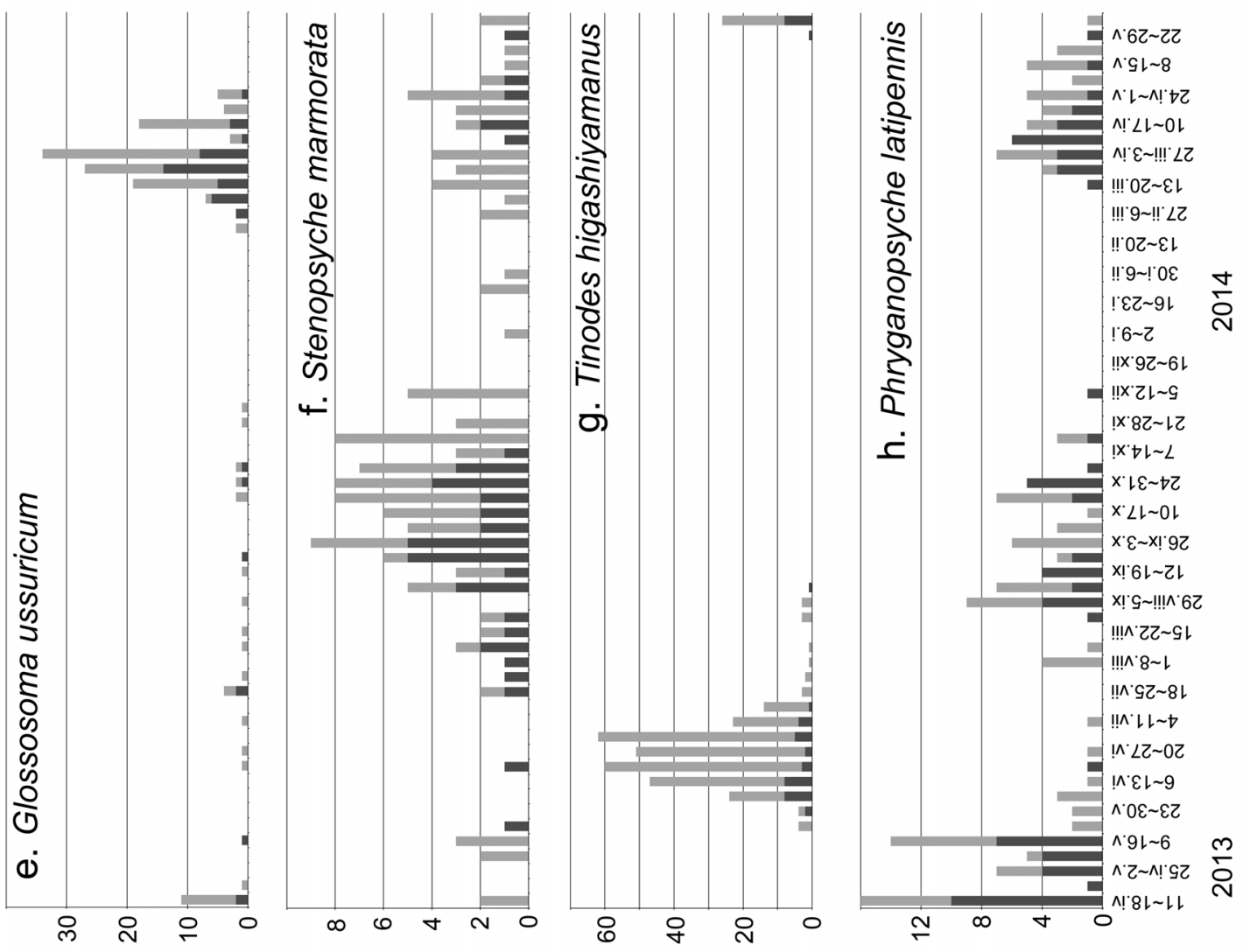

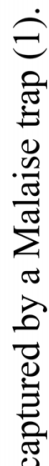
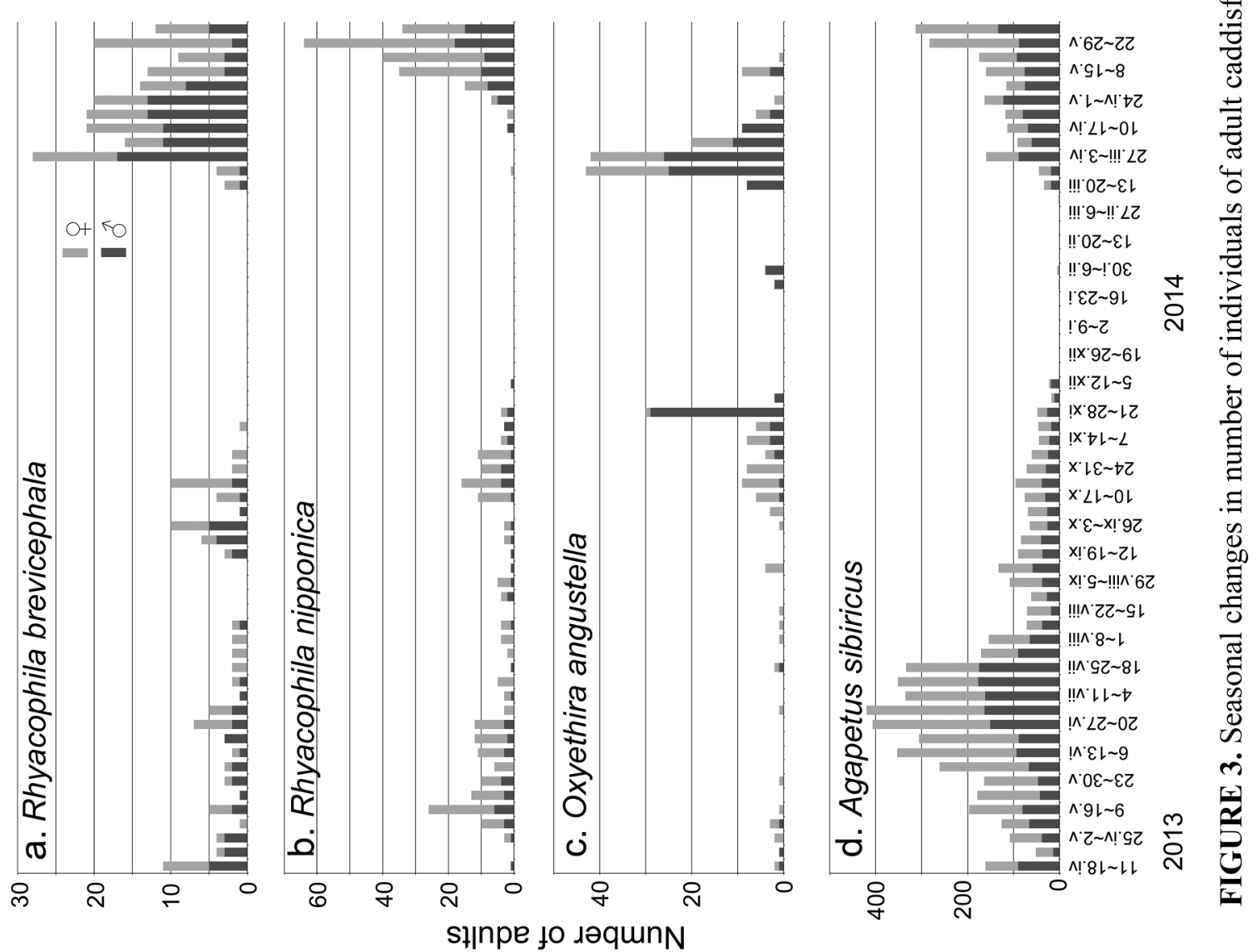

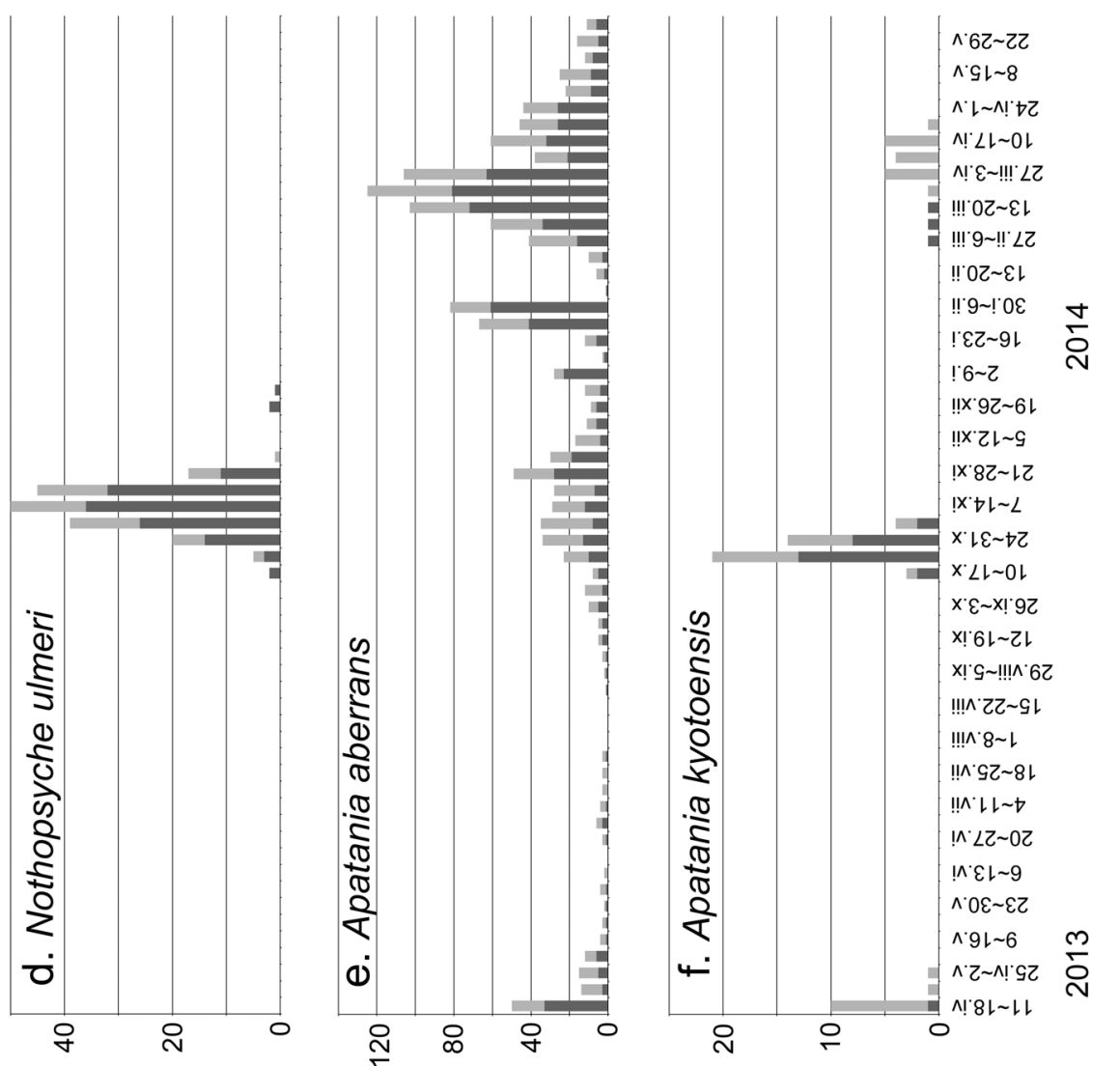

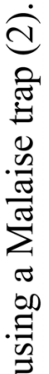
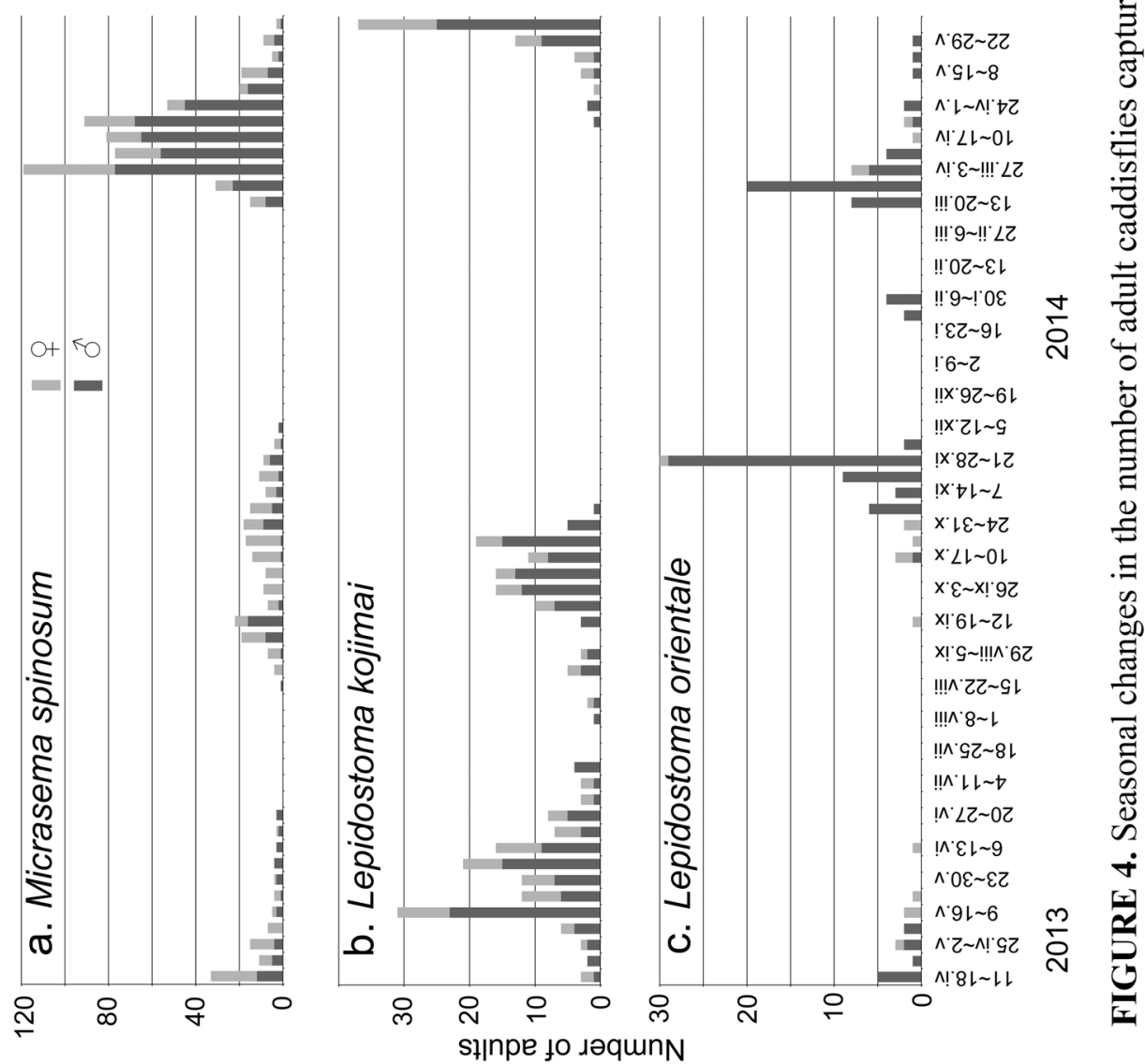
Tinodes higashiyamanus and Nothopsyche ulmeri have one narrow flight peak in early summer and midautumn, respectively (Figs. 3g, 4d). One short flight period in a year suggests that these species have a univoltine life cycle at the study site. $N$. ulmeri has a univoltine life cycle with a summer larval aestivation and autumn flight period (Nozaki 1989, as $N$. pallipes). The same autumn flight at the study site with constant water temperature must be regulated by the daily photoperiod.

Six species-Rhyacophila brevicephala, Rhyacophila nipponica, A. sibiricus, Glossosoma ussuricum, M. spinosum, and Lepidostoma kojimai-had one major peak in the spring or early summer (Figs. 3a, b, d, e, 4a, b), but their flight periods were extended throughout much of the year except for the winter. Furthermore, four of them-R. brevicephala, $R$. nipponica, M. spinosum, and L. kojimai-also had small autumn peak (Figs. 3a, $\mathrm{b}, 4 \mathrm{a}, \mathrm{b})$. These species have a univoltine life cycle with a spring or early summer flight period at the study site, but a proportion of the second generation that had rapidly grown probably emerge in autumn.

Apatania kyotoensis had two major peaks in the spring and autumn, and the period between the spring and autumn peak was longer than that between the autumn and spring peak (Fig. 4f). Nishimoto (1989) suggested that Japanese Apatania species originally had a univoltine life cycle with an autumn emergence period and that a proportion of the second generation that grew rapidly during winter could also emerge in the spring. He also suggested that they avoid high water temperatures during the summer season as they are at the prepupal stage during this season. The case of $A$. kyotoensis in this study supports his hypothesis. This species must aestivate during the summer, even with a constant water temperature, and has a second generation that emerges in the spring. On the other hand, Apatania aberrans had three peaks in the spring, autumn, and midwinter, and the former two peaks were broader than those of $A$. kyotoensis (Figs. 4e, f). This species probably has a more flexible life cycle and can emerge two or more times between the autumn and spring at the study site. Oxyethira angustella and Lepidostoma orientale had two major peaks in the spring and autumn, similar to $A$. kyotoensis, and they also had a small peak in the mid-winter (Figs. 3c, 4c). Although they may have a life cycle similar to that of $A$. kyotoenisis or $A$. aberrans, further studies, especially on the development of immature stages, are needed. In the Kakida River, adult $O$. angustella were collected throughout the year (Nozaki \& Tanida 2007, as O. kakida).

\section{Acknowledgements}

We express our thanks to Mr. Ikuro Hama, Mr. Toru Kohno, Mr. Yoshio Takashima, and all members of the Shimauchi Neighborhood Association in Matsumoto City for their valuable cooperation with respect to the installation and management of our Malaise trap. We are also indebted to Dr. Rie Saito, Mr. Kenji Mikami, Mr. JaeIck Jo, Mr. Ojiro Onishi and other members of the Tojo laboratory of Shinshu University for their cooperation with setting up the trap and specimen sampling and to Ms. Kimiko Iwakata, Kawasaki-shi, for the identification of bryophytes. This study was supported by a grant of the "Center of Community (COC)" program of the Ministry of Education, Culture, Sports, Science and Technology in Japan.

\section{References}

Banks, N. (1906) New Trichoptera from Japan. Proceedings of the Entomological Society of Washington, 7, 106-113.

Botosaneanu, L. (1970) Trichopteres de la Republique Democratique-Populaire de la Coree. Annales Zoologici, $27,275-359$.

Botosaneanu, L. (Ed.) (1998) Studies in crenobiology. The biology of springs and springbrooks. Backhuys Publishers, Leiden. $261 \mathrm{pp}$.

Ito, T., Kamei, H., Ohkawa, A., Kuhara, N. \& Nishimoto, H. (1998) Caddisfly fauna of the Shibetsu District and the Shiretoko Pass, easternmost part of Hokkaido, northern Japan. Biology of Inland Waters, 13, 1-17 [in Japanese with English abstract].

Ito, T. \& Kuhara, N. (2009) A new lotic species of the genus Leptocerus Leach (Trichoptera, Leptoceridae) from Japan. Limnology, 10, 25-31. https://doi.org/10.1007/s10201-008-0256-2

Iwata, M. (1927) Nihon-san moshi-moku yochu [Trichopterous larvae from Japan]. Zoological Magazine, Tokyo, 39, 209-272 [in Japanese]

Japan Meteorological Agency (2014) Japan Meteorological Agency Website. Available from: http://www.jma.go.jp/jma/ index.html (accessed 8 September 2014) 
Kobayashi, M. (1955) A new species of Dinarthrodes from Japan (Insecta: Trichoptera). Bulletin of the National Science Museum (Tokyo), 2, 70-72+ pl. 14.

Kobayashi, M. (1968) Notes on the caddisflies of Niigata Prefecture, with six new species. Bulletin of the Kanagawa prefectural Museum (Natural Science), 1 (1), 1-12 + pls. 1-6.

Kuhara, N. (2011) Species compositions and flight periods of caddisflies (Trichoptera) at headwater streams in Hokkaidô, northern Japan. Biology of Inland Waters, 26, 47-76. [in Japanese with English abstract]

Kumanski, K. (1990) Studies on the fauna of Trichoptera (Insecta) of Korea. I. Superfamily Rhyacophiloidea. Historia naturalis bulgarica, 2, 36-59.

Martynov, A.V. (1914) Notes on the Trichoptera collected by the Prof. P. Sushkin's Expedition to the Altai during 1912. Revue Russe d'Entomologie, 14, 72-84.

Martynov, A.V. (1917 [1918]) Sur une nouvelle espèce de la tribu des Apatniini et quelques autres formes provenant du pays de Minoussinsk. Annuaire du Musée Zoologique Académie Impériale des Sciences de Pétrograd, 22, 45-63. [in Russian]

Martynov, A.V. (1933) On an interesting collection of Trichoptera from Japan. Annotationes Zoologicae Japonenses, 14, 139 156.

Martynov, A.V. (1934) Tableaux Analytiques de la Faune de l'URSS, 13: Rucheyniki, Trichoptera, Annulipalpia, I. L'Institute Zoologique de l'Académie des Sciences, Leningrad, 343 pp.

Martynov, A.V. (1935) Trichoptera of the Amur region. Part I. Travaux de l'Institut Zoologique de l'Académie des Sciences de l'URSS, 2-3, 205-395.

Morisita, M. (1959) Measuring of interspecific association and similarity between communities. Memoires of the Faculty of Science, Kyushu University, Series E. Biology, 3, 65-80.

Navás, L. (1919 [1920]) Neurópteros (Ins.) del Japón. Revista de la Real Academia de Ciencias Exactas, Físicas y Naturales de Madrid, 18, 157-164.

Navás, L. (1933) Insecta Orientalia. Series 12. Memorie della Pontificia Accademia delle Scienze Nuovi Lincei, 17, 75-108.

Nishimoto, H. (1989) Koeguri-tobikera no shubunka to chiriteki bunpu [Speciation and geographical distribution of Apatania]. Shibatani, A. \& Tanida, K. (Eds.) Aquatic insects of Japan, Tokai Univ. Press, Tokyo, pp. 109-117. [in Japanese]

Nozaki, T. (1989) Hotaru-tobikera-zoku-Seikatsushi to bunpu [Nothopsyche-life history and distribution]. Shibatani, A. \& Tanida, K. (Eds.) Aquatic insects of Japan, Tokai Univ. Press, Tokyo, pp. 99-108. [in Japanese]

Nozaki, T. \& Tanida, K. (2007) The caddisfly fauna of a huge spring-fed stream, the Kakida River, in central Japan. BuenoSoria, J., Barba-Alvarez, R. \& Armitage, B. (Eds.), Proceedings of the 12th International Symposium on Trichoptera, The Caddis Press, Ohio, pp. 243-255.

Nozaki, T., Togashi, S. \& Sato, T. (2016) The caddisfly fauna of a small spring brook in the Jimoto-yusui, Niigata, central Japan. Vshivkova, T.S \& Morse, J.C. (Eds.) Proceedings of the 14th International Symposium on Trichoptera.

Ohkawa, A. (1999) Caddisfly fauna of a spring stream, Kikanko Creek, Tokachi, Hokkaido, northern Japan. Biology of Inland Waters, 14, 35-44 [in Japanese with English abstract]

Schmid, F. (1952) Le groupe de Chilostigma (Trichopt., Limnoph.). Archiv für Hydrobiologie, 47, 75-163.

Schmid, F. (1964) Quelques Trichoptéres Asiatiques. The Canadian Entomologist, 96, 825-840. https://doi.org/10.4039/Ent96825-6

Schmid, F. (1970) Le genre Rhyacophila et la famille des Rhyacophilidae (Trichoptera). Mémoires de la Société Entomologique du Canada, 66, 1- 230 + pls. 1-52. https://doi.org/10.4039/entm10266fv

Tani, K. (1971) A revision of the family Lepidostomatidae from Japan (Trichoptera). The Bulletin of the Osaka Museum of Natural History, 24, 45-70.

Townes, H. (1972) A light-weight Malaise trap. Entomological News, 83, $239-247$.

Tsuda, M. (1936) Untersuchungen über die japanischen Wasserinsekten II. Lepidostomatinae (Trichoptera). Annotationes Zoologicae Japonenses, 15, 400-409.

Tsuda, M. (1939) Zur Kenntnis der japanischen Apataniinen (Limnophilidae, Trichoptera). Annotationes Zoologicae Japonenses, 18, 290-293.

Tsuda, M. (1942) Japanische Trichopteren I. Systematik. Memoirs of the College of Science, Kyoto Imperial University, Series $B, 17,239-339$.

Ulmer, G. (1905) Zur Kenntniss aussereuropäischer Trichopteren. Stettiner Entomologishe Zeitung, 66, 3-119; pls. 1-4.

Ulmer, G. (1908) Japanische Trichopteren. Deutsche Entomologische Zeitschrift, 1908, 339-355. 